\title{
Valor nutricional de seis plantas arbóreo-arbustivas consumidas por cabras en la Mixteca Poblana, México
}

\section{Evaluation of six tree shrubs consumed by goats in Mixteca Poblana, Mexico}

\author{
${ }^{\bullet}$ Jorge Hernández Hernández ${ }^{1}$, Oscar Villarreal Espinobarros ${ }^{1}$, Julio Camacho Ronquillo ${ }^{1}$, \\ Salvador Romero Castañón ${ }^{1}$, Alberto de Jesús Castilloํㅜ José Lucio Hernández ${ }^{2}$ \\ ${ }^{1}$ Cuerpo Académico de Producción Animal de la FMVZ-BUAP, México. ${ }^{\circ}$ ovichiv_05@yahoo.com; \\ oscarvillarrealeb@hotmail.com; camacho90@colpos.mx; chavavetzoo@hotmail.com; alcaru_90@hotmail.com \\ ${ }^{2}$ Profesor Investigador de la Facultad de Medicina - Benemérita Universidad Autónoma de Puebla (BUAP), México. \\ ovichiv18@hotmail.com
}

\section{Resumen}

$\mathrm{E}$ n las comunidades de Maninalcingo y Tehuaxtla ubicadas en la región Mixteca, Puebla, México, se evaluó la composición nutricional de seis plantas arbóreo-arbustivas consumidas por el ganado caprino (Palo de Brasil, Tehuistle, Barba de chivo, Huamúchil, Tlaxistle negro y Cubata), en época lluviosa. Se analizaron tres unidades de producción familiar, donde se colectaron hojas, flores y frutos (vainas). Para comparar el promedio de bocados/hy la parte de la planta consumida por los caprinos se aplicó la prueba de Tukey $\mathrm{y}$, estadística descriptiva para determinar la dispersión entre los componentes bromatológicos de las partes de la planta de la población total de arbóreo-arbustivas evaluadas. Las muestras estuvieron constituidas por $200 \mathrm{~g}$ de peso verde (luego deshidratada). La materia orgánica $(\mathrm{MO})$, ceniza $(\mathrm{Ce})$ y proteína bruta $(\mathrm{PB})$ se obtuvieron por medio de la técnica AOAC (1997). Para el caso de la fibra detergente neutra (DDN), fibra detergente ácida (FDA) y lignina (Lig) por medio del método Van Soest. Los resultados demuestran que el Huamúchil (Pithecellobium dulce) mezclado con hoja y vaina mostró mejor media de PB $(18.30 \% \pm 1.08)$, la mejor PB fue para la fracción hoja $(14.40 \% \pm 0.42) \mathrm{de}$ Tehuistle (Acacia bilimekii var. Robusta Miranda) y $94.40 \% \pm 1.02$ en MO. El Huamúchil mezclado con hoja y vaina tuvo el promedio más alto de Ce (12.30 \pm 0.88$)$, mientras que la mezcla de vaina y cáscara de Cubata alcanzaron $52.70 \% \pm 1.80$ y $49.80 \% \pm 1.04$ de FDN y FDA, respectivamente. En el caso de lignina, la hoja de Tehuistle alcanzó $18.40 \% \pm 0.98$.

Palabras clave: nutrición, leñosas, composición, cabras, leguminosas.

Recibido: 27-septiembre-2014. Recibido en forma corregida: 28-noviembre-2014. Aceptado: 20-marzo-2015.

Publicado como ARTíCULO CIENTÍFICO en Ciencia y Tecnología 8(1): 19-23

Junio de 2015

\begin{abstract}
A $n$ evaluation of the nutritional composition of six tree shrubs (Palo de Brasil, Barba de chivo, Huamúchil, Tlaxistle negro y Cubata) consumed by goats was carried out In Maninalcingo and Tehuaxtla communities, located in Mixteca region, Puebla, Mexico, during the rainy season. Three family production units, where leaves, flowers and fruits were collected, were analyzed. In order to compare average hourly bite and the part of the plant consumed by goats Tukey test was applied together with descriptive statistics, the latter to determine the dispersion among bromatological components of the different parts of each plant in the total population of tree shrubs evaluated. The samples were constituted of 200 green weight grams (dehydrated after). Organic matter (MO), ash (Ce) and raw protein (PB) were obtained AOCA technique (1997). For the case of neutral detergent fiber (DDN), acid detergent fiber (FDA) and lignin (Lig.) Van Soest method was used. Results show that Huamúchil (Pithecellobium dulce) mixed with leaf and sheath had a better $\mathrm{PB}$ rate $(18.30 \% \pm 1.08)$, being the best PB rate $(14.40 \% \pm 0.42)$ for Tehuistle (Acacia bilimekii var. Robusta Miranda) leaf fraction and $94.40 \% \pm 1.02$ in MO. Huamuchil mixed with leaf and sheath showed also the highest rate of Ce (12.30 \pm 0.88$)$, while the mixture of Cubata sheath and rind achieved $52.70 \% \pm 1.80$ and $49.80 \% \pm 1.04$ of FDN y FDA, respectively. In the case of lignin, Tehuiste leaf achieved $18.40 \% \pm 0.98$.
\end{abstract}

Key words: nutrition, woody plants, composition, goats, legumes 


\section{Introducción}

$\mathrm{L}_{\mathrm{y}}^{\mathrm{a}}$ mayoría de las regiones áridas, semiáridas el trópico seco en el mundo, se encuentran pastoreadas por animales domésticos (bovinos, ovinos y caprinos), los cuales consumen con gran habilidad y dinamismo, las áreas ricas de arbóreas y arbustivas donde interactúan estos rumiantes con el componente leñoso (Hernández, 2006). Las especies arbóreas ofrecen diversos productos bioquímicos que en los animales puede producir interacciones difíciles de explicar con las formas tradicionales de estudiar los nutrientes de las plantas; un alto número de leñosas forrajeras, muestran potencialidades para ser utilizadas en el diseño de sistemas silvopastoriles (SSP) como estrategias para mitigar efectos de cambio climático y deficiencias nutricionales de rumiantes en zonas secas (Pinto et al., 2010).

En consecuencia, el follaje de arbustos y árboles se convierte en un recurso altamente estratégico del cual puede depender el mantenimiento de los animales en épocas críticas de disponibilidad de pasturas; además el consumo y la degradabilidad ruminal de materiales fibrosos dependen, entre otros factores, de sus características físicas (Giger-Reverdin, 2000), la ausencia de información relativa a variables como densidad, tamaño de partícula, capacidad de retención de agua y solubilidad es una limitante en la definición del valor nutricional de dichos materiales, lo cual es indispensable para el establecimiento de estrategias de manejo sostenible de sistemas Silvopastoriles (Korbut et al., 2009).

El uso de los recursos naturales, de forma racional y sostenible, es una opción viable para obtener beneficios en las actividades agropecuarias (FAO, 2012). Los árboles y arbustos forrajeros son una fuente inagotable de nutrientes, que aportan alimentos de buena calidad, la mayor parte del año, mejora la dieta del animal y reduce el uso de concentrados en las explotaciones pecuarias (De Andrade et al., 2008 y Ortega, 2012).

Las arbóreo-arbustivas en la Mixteca poblana consumidas por el ganado caprino, son predominantemente el potencial forrajero más importante en las dietas de estos rumiantes; integradas principalmente por leguminosas (Hernández et al., 2005; Villarreal, 2006). Las leguminosas, por su gran versatilidad y diversas bondades, han jugado un papel muy importante por mucho tiempo en los sistemas agroforestales y silvopastoriles en el trópico (Portillo et al., 2009) y en la selva baja caducifolia de la Mixteca Poblana (Arroyo et al., 2007).

Hay que puntualizar que ese potencial forrajero, tiene diferentes partes vegetativas como son: hojas, flores y frutos, con diferentes porcentajes de componentes nutricionales; lo cual hace, un importante forraje con diferentes valores nutricionales, según sea la parte consumida y la época en que se encuentre este banco de proteína natural durante el año (Orskov, 2005; Hernández et al., 2008). El valor nutritivo de los alimentos está dado por su composición química y por la eficiencia con que los animales extraen sus nutrientes durante la digestión (Sanon et al., 2008). Por esta razón, es importante conocer el contenido de nutrientes y el valor nutricional de especies individuales nativas (Guerrero et al., 2010). El objetivo del estudio fue conocer el valor nutricional de seis plantas arbóreo-arbustivas que consumen en una época del año los caprinos en la Mixteca Poblana.

\section{Materiales y métodos}

\section{Localización del estudio}

Se realizó en las comunidades de Maninalcingo y Tehuaxtla pertenecientes al municipio de Piaxtla, se ubican en la región Mixteca del sureste del estado de Puebla, localizándose entre los paralelos $17^{\circ} 59^{\prime} 00^{\prime \prime}$ y $18^{\circ} 12^{\prime} 30^{\prime \prime}$ latitud norte y los meridianos $98^{\circ} 10^{\prime}$ 54 " y $98^{\circ} 21^{\prime} 36^{\prime \prime}$ longitud oeste. El área de estudio, cuenta con terrenos accidentados y altitudes variadas que van de los 700 a los 2000 msnm (Gómez et al., 1996). En lo que respecta a su flora, está integrada por selva baja caducifolia, selva baja caducifolia espinosa, vegetación xerófita y vegetación secundaria, localizándose pequeñas áreas de bosque de encino y pastizales. La fauna silvestre está representada principalmente por venado cola blanca (Odocoileus virginianus), coyote (Canis latrans), zorrillo (Mephitis macroura), armadillo (Dasypus novemcinctus), iguana (Ctenosaura pectinata) y serpiente de cascabel (Crotalus spp.), entre otras especies (Villarreal, 2000). El clima es cálido subhúmedo con lluvias en verano que van de los 350 a los $800 \mathrm{~mm}$, y semiseco muy cálido. La temperatura media anual es de $23^{\circ} \mathrm{C}$. El estudio se desarrolló durante cinco meses en la época de lluvia de junio a noviembre del 2004.

\section{Colección y procesamiento de las muestras}

Las muestras se colectaron entre las $8.30 \mathrm{am}$ y $12.30 \mathrm{pm}$. Se utilizaron 3 unidades de producción familiar (UPF) caprinas. Se identificaron 5 caprinos/ UPF para el estudio y selección de la planta ramoneada, de acuerdo a la preferencia y número de bocados dados, especialmente a la parte(s) consumidas por los caprinos, a una altura que no sobrepasara los $2 \mathrm{~m}$ sobre el nivel del suelo de las UPF caprinas; se colectó la planta, anotándo en una base de datos 
(nombre común o vulgar), todo esto apoyado con el pastor-guía para el reconocimiento del componente leñoso (arbóreo-arbustivo) observado, a través del método de observación directa durante el periodo de estudio en la región Mixteca; posteriormente la planta colectada (ramilla) de 20 a $25 \mathrm{~cm}$ con hoja, flor y fruto (vainas verdes y secas) se colocó en una tabla-prensa y se separó con papel periódico para su transportación, protección y clasificación científica (nombre) al herbario de la Escuela de Biología de la BUAP.

Se empleó tijeras de jardinería para el corte de la ramilla y cámara digital para la obtención del material fotográfico del componente leñoso en estudio. En cada una de las 3 unidades de producción familiar, la colección del material (partes de la planta consumida) fue de tres árboles o arbustos como mínimo, constituyéndose una muestra compuesta de $200 \mathrm{~g}$ para su análisis en el laboratorio.

Las muestras se secaron a $35^{\circ} \mathrm{C}$ durante $72 \mathrm{~h}$ en una estufa con circulación forzada de aire; posteriormente se molieron con un molino de martillo hasta un tamaño menor de $1 \mathrm{~mm}$, los análisis químicos de materia orgánica $(\mathrm{MO})$, ceniza $(\mathrm{Ce})$ y proteína bruta $(\mathrm{PB})$ se valoraron siguiendo las técnicas según la AOAC (1997). Mientras que para las determinaciones de fibra detergente neutra (FDN), fibra detergente ácido (FDA) y lignina (Lig) se realizaron por el método de Van Soest (1994). Análisis desarrollados en el Laboratorio de Bromatología del Colegio de Posgraduados de Montecillos, Estado de México.

Se aplicó prueba de Tukey simple (ANAVA) para la preferencia y parte de la planta consumida por los caprinos, y estadística descriptiva para la composición bromatológica, fracción vegetativa de las plantas consumidas. Se realizó 10 repeticiones para cada fracción de la planta y se obtuvo la Media \pm Desviación estándar $(\mathrm{M} \pm \mathrm{DE})$, utilizando el paquete SPSS 14.0 para Windows.

\section{Resultados y discusión}

$\mathrm{L}$ a composición botánica de la dieta para caprinos en la región Mixteca durante la época de lluvias, permitió identificar 40 plantas arbóreo-arbustivas; a través del método de observación directa en las tres UPF caprinas consideradas en el estudio; de las cuales correspondió a la familia de las Mimosoidaes (leguminosas), similar resultado encontró Villarreal et al. (2008) con el 37.5\% de leguminosas, en siete Unidades de Manejo Ambiental (UMA's) de cinco municipios de la Mixteca Poblana, con 133 plantas consumidas por venados y caprinos, a través de los métodos de observación directa y microhistológico. De esta composición leñosa, sobresalen seis arbóreoarbustivas consumidas y preferidas (bocados dados) por el ganado caprino observado, en las tres UPF de Tehuaxtla y Maninalcingo (Cuadro 1).

El consumo de la parte foliar es considerado y representa una importante fuente continua y de alto valor nutricional, por la proteína aportada por las leguminosas del componente leñoso preferido y consumido por las cabras de la región Mixteca; resultados similares, encuentran Franco et al. (2005) y Hernández (2006) durante todo el periodo de crianza de los caprinos, en la región de la Mixteca Oaxaqueña y Poblana al preferirlo y consumirlo. La parte consumida (bocados/h/dados a la parte o fracción) de las plantas consideradas para su composición bromatológica, presentan diferencias en las hojas de todas las arbóreoarbustivas, a excepción de la cubata blanca (Cuadro 2).

Es importante resaltar, que la calidad de la dieta que consume el caprino mixteco, dependerá de la condición del estrato arbóreo-arbustivo, la capacidad de carga del hábitat y manejo del pastoreo; así, como de las densidades poblacionales de otras especies animales (bovinos, ovinos e incluso venados) presentes en la región, estación del año, precipitación y la composición

Cuadro 1. Nombres y familias científicas de arbóreo-arbustivas que muestran la parte o fracción consumida por los caprinos de la Mixteca Poblana

\begin{tabular}{lllll}
\hline Nombre común & Nombre científico & Familia taxonómica & $\begin{array}{l}\text { Tipo de } \\
\text { planta }\end{array}$ & $\begin{array}{l}\text { Parte } \\
\text { consumida }\end{array}$ \\
\hline Palo de Brasil & Haemotoxylumbrasiletto & Caesalpinioideae & Arbórea & Hoja \\
Tehuistle & Acacia bilimekii var. Robusta Miranda & Mimosoidae & Arbustiva & Hoja \\
Barba de Chivo & Pithecellobium acatlense & Mimosoideae & Arbórea & Hoja y vaina \\
Huamúchil & Pithecellobium dulce & Mimosoideae & Arbórea & Hoja y Vaina \\
Tlaxistle Negro & Amelanchir denticulada & Rosaceae & Arbórea & Hoja \\
Cubata & Acacia pennatula. & Mimosoideae & Arbustiva & Vaina y cáscara \\
\hline
\end{tabular}


Hernández et al., 2015

Cuadro 2. Bocados/h dados a la parte o fracción en las plantas consumida por las cabras de las UPF en la región Mixteca

\begin{tabular}{lccccc}
\hline \multirow{2}{*}{ Plantas } & \multicolumn{3}{c}{ Partes de la planta } & \multirow{2}{*}{ ES } & Significancia \\
\cline { 2 - 4 } & Flor & Fruto & Hoja & & $*$ \\
\hline Palo de Brazil & $6.00 \mathrm{a}$ & $7.00 \mathrm{~b}$ & $34.20 \mathrm{a}$ & 3.37 & $*$ \\
Barba de Chivo & $0.00 \mathrm{c}$ & $9.50 \mathrm{~b}$ & $39.00 \mathrm{a}$ & 3.84 & NS \\
Cubata & 0.00 & 10.60 & 0.00 & 2.18 & $*$ \\
Huamuchil & $0.00 \mathrm{~b}$ & $21.50 \mathrm{~b}$ & $34.20 \mathrm{a}$ & 3.61 & $*$ \\
Tehuistle & $0.00 \mathrm{c}$ & $16.20 \mathrm{~b}$ & $31.30 \mathrm{a}$ & 3.71 & $*$ \\
Tlaxistle Negro & $0.00 \mathrm{c}$ & $6.38 \mathrm{~b}$ & $30.30 \mathrm{a}$ & 3.53 & $*$
\end{tabular}

Cuadro 3. Composición bromatológica de las principales fracciones y plantas consumidas por el ganado caprino durante la época de lluvia en la Mixteca Poblana $(\mathrm{M} \pm \mathrm{DE})$

\begin{tabular}{lcccccc}
\hline Planta y fracción consumida & MO & Ce & PB & FDN & FDA & Lig \\
\hline Palo de Brazil (hoja) & $89.90 \pm 0.88$ & $10.20 \pm 0.08$ & $12.30 \pm 0.80$ & $38.90 \pm 0.09$ & $22.60 \pm 0.64$ & $5.40 \pm 0.08$ \\
Tehuistle (hoja) & $94.40 \pm 1.02$ & $5.60 \pm 0.12$ & $14.40 \pm 0.42$ & $50.30 \pm 0.92$ & $39.60 \pm 0.98$ & $18.40 \pm 0.98$ \\
Barba de Chivo (hoja y vaina) & $90.90 \pm 0.86$ & $9.10 \pm 0.42$ & $14.80 \pm 0.44$ & $34.90 \pm 0.98$ & $22.50 \pm 0.22$ & $5.40 \pm 0.12$ \\
Huamúchil (hoja y vaina) & $87.70 \pm 0.94$ & $12.30 \pm 0.88$ & $18.30 \pm 1.08$ & $35.30 \pm 0.09$ & $25.10 \pm 0.26$ & $8.20 \pm 0.20$ \\
Tlaxistle Negro (hoja) & $91.10 \pm 1.08$ & $8.90 \pm 0.09$ & $13.10 \pm 0.62$ & $30.90 \pm 0.6$ & $17.70 \pm 0.08$ & $6.40 \pm 0.64$ \\
Cubata (vaina y cáscara) & $94.10 \pm 0.84$ & $5.90 \pm 0.08$ & $9.30 \pm 0.52$ & $52.70 \pm 1.8$ & $49.80 \pm 1.04$ & $15.90 \pm 0.08$ \\
\hline
\end{tabular}

de especies vegetales en el hábitat; sin embargo, el valor nutricional dependerá de la integración suelo planta-animal, más que de la selectividad y número de plantas que puedan integrar el ecosistema (Arroyo et al., 2007). El Cuadro 3 muestra la Media \pm Desviación estándar $(\mathrm{M} \pm \mathrm{DE})$ de la composición bromatológica, en la fracción o parte de las principales plantas consumidas por el ganado caprino durante la época de lluvia en la región Mixteca de Puebla.

\section{Conclusiones}

E 1 conocimiento de los componentes nutricionales que integran las arbóreo-arbustivas consumidas por el ganado caprino y otras especies domesticas (bovinos y ovinos), incluyendo la fauna silvestre como el venado cola blanca en la Mixteca Poblana, es un importante indicador en la alimentación de estos pequeños rumiantes; ya que, la proteína de las seis plantas leñosas valoradas alcanzaron un promedio de $13.70 \%$, siendo superiores a otros forrajes como los pastos nativos de esa región. De tal forma, el análisis químico es una importante valoración para conocer el potencial nutricional en la Mixteca 'Poblana de México, para la producción de caprinos durante la época de lluvias; sin embargo, es esencial y recomendable valorar este potencial en la época seca para tener mejor conocimiento nutricional durante todo el año.

\section{Bibliografía}

AOAC. 1997. (Association of Official Analytical Chemists). Official methods of analysis. 17th ed., William Horwitz editor, Washington D.C., USA.

Arroyo, H.S., Franco, G.F.J., Villarreal, EB.O., Reséndiz, M.R., Hernández, H.J.E. 2007. Clasificación taxonómica del potencial forrajero de tipo arbóreo-arbustivo consumido por el ganado caprino en el municipio de Piaxtla perteneciente a la Mixteca Poblana. Memoria in extenso CDR. XXII Reunión Nacional sobre Caprinocultura. Zacatecas, México. Pp. 25 
De Andrade, C.M.S., Valentín, J.F., Carneiro, J. Vaz, F.A. 2008. Growth of tropical forage grasses and legumes under shade. Pesquisa Agropecuária Brasileira 39:263

FAO. 2012. Use of lesser-known plants and plant parts as animal feed resources in tropical regions, by Emmanuel S. Quansah \& Harinder P.S. Makkar. Animal Production and Health Working Paper. No. 8. Rome

Franco, G.F.J., Gómez, G., Mendoza, G., Bárcena, R., Ricalde, R., Plata. F., Hernández, H.J.E. 2005. Influence of plant cover on dietary selection by goats in the Mixteca region of Oaxaca, México. Applied Animal Research 27: 95-100.

Giger-Reverdin, S. 2000. Characterization of feedstuffs for ruminants using some physical parameters. Animal Feed Science and Technology 86: 53

Gómez, Q.J.M., Amaro, G., Preciado de la Torre, G., Martínez, L. 1996. Marco de referencia para la Caprinocultura de la Mixteca Poblana. Memoria in extenso CD-R. XI Reunión Nacional sobre Caprinocultura. Chapingo, México. Pp.19.

Guerrero, M., Juárez, A.S., Ramírez, R.G., Montoya, R., Murillo, M., Lao, O., Cerrillo, M.A. 2010. Composición química y degradabilidad de la proteína de forrajes nativos de la región semiárida del norte de México. Revista Cubana de Ciencia Agrícola 44(2): 147-154.

Hernández, H.J.E. 2006. Valoración de la caprinocultura en la Mixteca Poblana: socioeconomía y recursos arbóreo-arbustivos. [Tesis Doctoral]. Universidad de Camagüey, Cuba.

Hernández, H.J.E., Franco, G.F.J., Contreras, J.L., Pedraza, R., Zamitiz, J., Herrera, G. 2005. Identificación de las principales plantas forrajeras de tipo arbóreo y arbustivo consumidas por caprinos en la Mixteca Poblana. Memorias in extenso CDR. XXIX Congreso Nacional de Buiatria. Puebla, México. Pp. 35.

Hernández, J.E., Franco, G.F.J., Villarreal, EB.O., Aguilar, L.M., Sorcia, M.G. 2008. Identificación $\mathrm{y}$ preferencia de especies arbóreo-arbustivas $\mathrm{y}$ sus partes consumidas por el ganado caprino en la Mixteca Poblana, Tehuaxtla y Maninalcingo, México. Zootecnia Tropical 26(3): 379-382.

Korbut, N., Ojeda, A., Muñoz, D. 2009. Evaluación del perfil bromatológico y de algunos parámetros físicos del follaje de plantas leñosas consumidas por vacunos en silvopastoreo en un bosque seco tropical semideciduo. Zootecnia Tropical 27(1): 65-72.
Orskov, E.R. 2005. Silvopastoral systems: technical, enviromental and socio-economic challenges. Rev. Est. Experiment. Pastos y Forrajes 28(1): 5-9.

Ortega, E. 2012. Potencial productivo de Guazuma ulmifolia Lam. en bancos de forraje y asociados a gramíneas tropicales. Tésis Maestría, Colegio de posgraduados Campus Veracruz, Veracruz, México, $110 \mathrm{p}$.

Pinto, R; Hernández, D; Gómez, H; Cobos, MA; Quiroga, R; Pezo, D. 2010. Árboles forrajeros de tres regiones ganaderas de Chiapas, México. Usos y características nutricionales. Universidad y Ciencia 26(1):19-31.

Portillo, A.B., Razz-García, R.C., Marín, M., AraujoFebres, O. 2009. Dinámica de crecimiento en plantas de añil dulce (Indigofera hirsuta L.). Archivos Latinoamericanos de Producción Animal 17(3-4): 91-96.

Sanon, H.O., Kabore-Zoungrana, C., Ledin, I. 2008. Nutritive value and voluntary feed intake by goats of three browse fodder species in the Sahelian zone of West Africa. Animal Feed Science and Technology 144: 97.

Van Soest, P.J. 1994. Nutritional Ecology of the Ruminnant. 2th edition. Cornell University Press. Ithaca, N. Y., USA. 115 pp.

Villarreal, O. 2000. Aprovechamiento sustentable del venado Cola Blanca Mexicano (Odocoileus, virginianus mexicanus). Una alternativa para el uso del suelo en la región de la Mixteca Poblana. [Tesis Maestría]. Universidad Autónoma de Tlaxcala, México.

Villarreal, O. 2006. Conservación y manejo del venado cola blanca mexicano (Odocoileus, virginianus mexicanus) como propuesta del modelo de ganadería diversificada en la Mixteca Poblana, México. [Tesis Doctoral]. Universidad de Camagüey, Cuba.

Villarreal, O., Cortes, M.I., Guevara, V.R., Franco, F.J., Campos, A., Castillo, J.C. 2008. Conservación y Manejo de Fauna Cinegética de México I, En: Villarreal, O., Hernández, HJE., Franco, FJ., Camacho, JC. (Editores). Composición botánica de la dieta del venado cola blanca (Odocoileus virginianus) en la Mixteca Poblana. Primera edición. Sierra, Puebla, México. 34-42 pp. 